\title{
miR-497 suppresses angiogenesis in breast carcinoma by targeting HIF-1 $\alpha$
}

\author{
ZHIHAO WU ${ }^{1 *}$, XUEHONG CAI ${ }^{2}, \mathrm{CHENGGANG} \mathrm{HUANG}^{2}, \mathrm{JIA} \mathrm{XU}^{2}$ and AN LIU ${ }^{2 *}$ \\ ${ }^{1}$ The Second Affiliated Hospital and Yuying Children's Hospital of Wenzhou Medical University, Wenzhou, Zhejiang 414000; \\ ${ }^{2}$ The First People's Hospital of Yueyang, Yueyang, Hunan 325000, P.R. China
}

Received October 7, 2015; Accepted November 11, 2015

DOI: $10.3892 /$ or.2015.4529

\begin{abstract}
Angiogenesis is a key factor in the growth and dissemination of malignant diseases, including breast cancer, with significant implications for its clinical management. It is known that microRNAs (miRNAs) play important roles in regulating tumor properties in cancers. However, whether miR-497 contributes to breast cancer angiogenesis remains unknown. Our study demonstrated that miR-497 was significantly downregulated in breast cancer tissue samples and cell lines. Conditioned medium obtained from breast cancer cell line MCF-7, treated with miR-497 mimics, suppressed the proliferation and tube formation of human umbilical vein endothelial cells in vitro, in comparison with the untransfected cells or cells transfected with the control vector alone. Furthermore, western blot assay confirmed that the overexpression of miR-497 reduced VEGF and HIF-1 $\alpha$ protein levels. In addition, stable transfection of miR-497 inhibited tumorigenicity and angiogenesis in vivo. Moreover, HIF-1 $\alpha$ was also increased in the breast cancer cells under a hypoxic condition, while the ectopic expression of miR-497 partially restored its level. Taken together, our findings indicate that miR-497 is a potential target for the biological therapy of breast cancer. Moreover, miR-497 inhibited the growth of
\end{abstract}

Correspondence to: Dr An Liu, Department of General Surgery, The First People's Hospital of Yueyang, 39 Dong Mao Ling Road, Yueyang, Hunan 325000, P.R. China

E-mail: hosrml@qq.com

${ }^{*}$ Contributed equally

Abbreviations: miRNAs, microRNAs; VEGF, vascular endothelial growth factor; HIF-1 $\alpha$, hypoxia inducible factor-1 $\alpha$; HUVECs, human umbilical vein endothelial cells; ATCC, American Type Culture Collection; NC, negative control; CCK-8, Cell Counting Kit-8; RT-PCR, real-time polymerase chain reaction; PBS, phosphate-buffered saline; MVD, microvessel density; HRP, horseradish peroxidase; TUNEL, terminal deoxynucleotidyl transferase-mediated dUTP nick-end labeling

Key words: breast cancer, miR-497, angiogenesis, VEGF, HIF-1 $\alpha$ tumors and reduced angiogenesis in a nude mouse xenograft tumor model, which was probably caused by the downregulation of pro-angiogenic molecules, such as VEGF and HIF-1 $\alpha$.

\section{Introduction}

Currently, breast cancer is the most prevalent oncological disease worldwide, with approximately 232,670 new cases and 40,000 deaths among women living in the United States in 2012 (1). The most aggressive and invasive tumor cells, including breast cancer cells, which often reside in hypoxic environments, rely on extensive glycolysis to meet their large demand for energy and biosynthetic precursors $(2,3)$. Once exposed to a hypoxic environment, breast cancer cells subsequently exhibit enhanced angiogenesis and tumor metastasis, which results in the poor survival rate of cancer patients $(4,5)$. Hence, investigation of hypoxia-induced molecules will aid in the development of new tools for the treatment of breast cancer.

MicroRNAs (miRNAs) are a type of endogenous and small non-coding regulatory RNAs of 21-23 nucleotides, which mainly recognize complementary sequences in the 3'-untranslated regions (UTRs) of their target genes leading to mRNA degradation or translation inhibition. Most animal miRNAs are evolutionarily conserved and are often found in clusters $(6,7)$. Primary miRNAs with a stem-loop structure are transcribed by RNA polymerases (RNA Pols) and processed in both the nucleus and cytoplasm by Drosha as well as Dicer to generate mature miRNAs (8). Existing studies have found that miRNAs are involved in the regulation of multiple pathological processes that contribute to tumorigenesis and metastasis, such as tumor cell proliferation, differentiation, apoptosis, as well as invasion (9). For example, a higher level of miR-191 was reportedly found in several types of cancer, including breast cancer, in which it was found to be hypoxia inducible (10). In addition, miRNA-10b (miR-10b) expression was increased in metastatic breast cancer cells, along with positive regulation of cell migration and invasion through the suppression of the homeobox D10 tumor-suppressor signaling pathway (11). In most solid cancers, miR-497 has been found to be a tumor suppressor (12-14). Such a function is exerted partly by anti-proliferative and anti-growth potential (13-15). A previous study demonstrated that miR-497 induced cell apoptosis by negatively regulating $\mathrm{Bcl}-2$ protein expression 
at the post-transcriptional level in human breast cancer (13). However, the relationship between miR-497 and hypoxia remains unclear.

In the present study, we found that miR-497, as a hypoxia responsive miRNA, was downregulated in human breast cancer clinical samples and cell lines. Ectopic expression of miR-497 functioned as an angiogenesis suppressor both in vitro and in nude mice by targeting VEGF and HIF-1 $\alpha$. Our results propose that miR-497 may be a therapeutic target for breast cancer in the development of angiogenesis inhibitors.

\section{Materials and methods}

Patients and tissue specimens. Forty-five human breast cancer clinical samples and their corresponding normal breast tissues were obtained from breast cancer patients who underwent surgery for breast cancer at The Second Affiliated Hospital of Wenzhou Medical University between October 2013 and October 2014. All cases were histologically confirmed as invasive, ductal breast cancer by trained pathologists. No patients received chemotherapy or radiotherapy prior to surgery. All the specimens were obtained with informed consent and approved by the Ethics Committee of Wenzhou Medical University.

Cell culture. Human breast cancer cell lines T-74D, MCF-7, MDA-MB-453, MDA-MB-468 and MDA-MB-435 were purchased from the American Type Culture Collection (ATCC; Rockville, MD, USA). Non-malignant breast epithelial cell line MCF-10A was preserved in our laboratory. The cells were cultured in modified Eagle's medium containing $10 \%$ fetal bovine serum (FBS), $0.5 \%$ penicillin-streptomycin, and $1 \%$ glutamine at $37^{\circ} \mathrm{C}$ with $5 \% \mathrm{CO}_{2}$. HUVECs (obtained from ATCC) were cultured in gelatin-coated plates with M199 medium containing $20 \%$ FBS, endothelial cell growth supplement $\left(50 \mu \mathrm{g} / \mathrm{ml}\right.$; Sigma) and antibiotics, and incubated at $37^{\circ} \mathrm{C}$ in $5 \% \mathrm{CO}_{2}$ in air. For hypoxic stress, MCF-7 cells were placed in a hypoxia chamber (Invivo200; Ruskin, UK) maintained at different $\mathrm{pO}_{2}$ conditions (ranging from 0.2 to $1 \%$ oxygen) and $5 \% \mathrm{CO}_{2}$. Cells in the logarithmic growth phase ( $\sim 90 \%$ confluency) were selected for the experiments.

RNA transfection. miR-497 mimics, inhibitors (anti-miR-497) and AllStar negative control siRNA (NC) were synthesized by GenePharma (Shanghai, China). The transfection was conducted using Lipofectamine 2000 (Invitrogen, USA) according to the manufacturer's instructions. Complete medium was changed $5 \mathrm{~h}$ after transfection.

Preparation of tumor cell-conditioned medium. After MCF-7 cells were transfected for $48 \mathrm{~h}$, the supernatant medium was replaced by serum-free medium and incubation was carried out for another $24 \mathrm{~h}$. The conditioned medium was collected after centrifugation at $4^{\circ} \mathrm{C}$ and $2,000 \mathrm{rpm}$ for $10 \mathrm{~min}$ and was stored at $-70^{\circ} \mathrm{C}$ for subsequent use. The cells were classified as the negative control siRNA conditioned medium group (NC), the miR-497 mimics conditioned medium group and the antimiR-497 conditioned medium group, respectively.

Cell proliferation. HUVEC cells were seeded in each well of 96-well culture plates (1,000 cells/well). After overnight incubation, the medium was removed and replaced with fresh culture medium plus equal amounts of the different conditioned medium. After $48 \mathrm{~h}$ of incubation, the supernatant was discarded, and $10 \mu \mathrm{l}$ CCK-8 solution (Dojin Laboratories, Kumamoto, Japan) was added. The cells were then incubated at $37^{\circ} \mathrm{C}$ for $60 \mathrm{~min}$, and the absorbance was measured at $490 \mathrm{~nm}$ using a microplate spectrophotometer (Bio-Tek, USA). This experiment was repeated twice.

Quantitative real-time PCR (RT-PCR). Total RNA extraction was performed using TRIzol reagent (Life Technologies, Carlsbad, CA, USA) according to the manufacturer's instructions. Real-time qPCR was used to confirm the expression levels of mRNAs. cDNA was produced according to the protocol for PrimeScript ${ }^{\mathrm{TM}}$ RT reagent (Takara, Japan). The expression levels of miRNAs were analyzed using TaqMan MicroRNA Assay kits (Applied Biosystems, Foster City, CA, USA). Data analysis was performed using the $2^{-\Delta \Delta C t}$ method. All experiments were performed in triplicate.

Tube formation assay. For the capillary tube formation assay, Matrigel (Becton Dickinson, USA) was dissolved at $4^{\circ} \mathrm{C}$ overnight, and each well of prechilled 96 -well plates was coated with $100 \mu 1$ Matrigel and incubated at $37^{\circ} \mathrm{C}$ for $45 \mathrm{~min}$. HUVECs were transferred to the 96-well plates with the different conditioned medium at the density of $1 \times 10^{4} /$ well for $12 \mathrm{~h}$ in a humidified $5 \% \mathrm{CO}_{2}$ atmosphere. Capillary-like structures of HUVECs were photographed and the light micrograph images were stored in a computer. Tubular structures were quantified by manual counting at x100 magnification.

Western blot analysis. Cells were washed three times with ice-cold PBS and lysed in NP-40 lysis buffer [20 mmol/1 Tris- $\mathrm{HCl}$ (pH 7.4), $100 \mathrm{mmol} / 1 \mathrm{NaCl}, 1 \% \mathrm{NP}-40,0.5 \%$ sodium deoxycholate, $5 \mathrm{mmol} / 1 \mathrm{MgCl}_{2}, 0.1 \mathrm{mmol} / \mathrm{l}$ phenylmethylsulfonyl fluoride and $10 \mathrm{mg} / \mathrm{ml}$ of protease inhibitor mixture]. Protein was extracted using Mammalian Protein Extraction reagent and its concentration was determined by BCA assay (both from Pierce Inc., Rockford, IL, USA). Total proteins (20-40 $\mu \mathrm{g}$ ) from each sample were electrophoresed on $8 \%$ SDS-PAGE gel, and transferred to a nitrocellulose membrane. The membranes were blocked in 5\% nonfat milk and probed with the primary antibodies as indicated overnight at $4^{\circ} \mathrm{C}$, and then with the respective secondary antibodies. Band signals were visualized using an enhanced chemiluminescence kit (Pierce, Minneapolis, MN, USA). The same membrane was reprobed with the anti- $\beta$-actin antibody, which was used as the internal control.

In vivo tumorigenesis. In vivo experiments were conducted as described previously (16). Briefly, we used male athymic $\mathrm{BALB} / \mathrm{c}$ nu/nu mice (4-6 weeks old) that were maintained in standard mice plexiglass cages in a room maintained at constant temperature and humidity under a $12 \mathrm{~h}$ light and darkness cycle. A total of $1 \times 10^{7} \log$ arithmically growing cells from the different groups: a) MCF-7 cells; b) MCF-7 cells transfected with the negative control; c) MCF-7 cells transfected with the miR-497 mimics were injected subcutaneously into the midabdominal area, respectively. After 40 days of observation, the mice were sacrificed and tumors were surgically excised neatly 
A

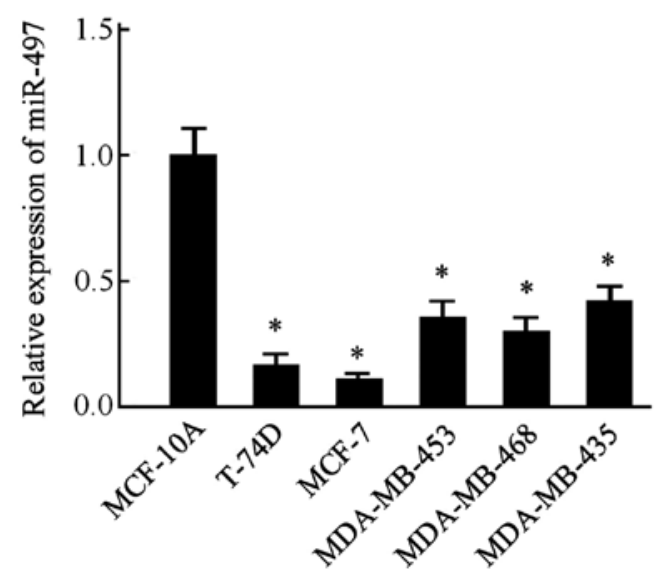

B

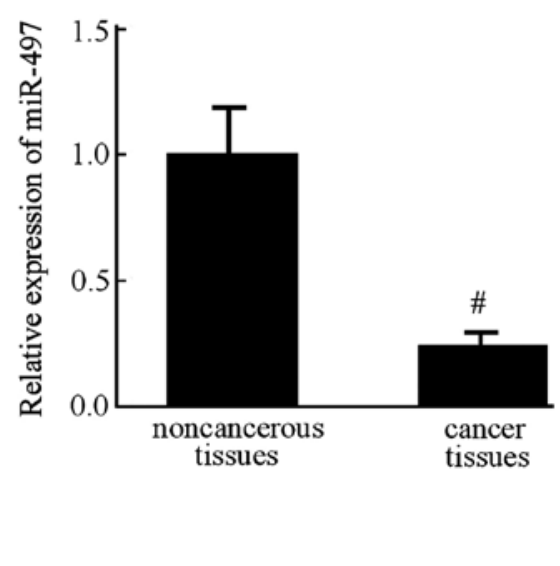

Figure 1. Reduced expression of miR-497 in breast cancer cells and tissues. (A) The expression of miR-497 was downregulated in five human breast cancer cell lines compared to its level in non-malignant breast epithelial cell line MCF-10A. (B) Relative miR-497 expression was downregulated in breast cancer tissues compared with the level in matched adjacent non-cancerous breast tissues. ${ }^{*} \mathrm{P}<0.05$ vs. MCF-7 cell group, ${ }^{\text {\# }} \mathrm{P}<0.05$ vs. non-cancerous tissue group.

and weighed. One part of the tissue was fixed in formalin and another part was frozen in liquid nitrogen.

Immunohistochemical and microvessel density (MVD) evaluation. Xenograft tumor samples were embedded in paraffin and fixed with paraformaldehyde. After being washed in PBS, the slides were blocked with protein block solution (DakoCytomation) to block endogenous peroxidase activity. Such samples were then incubated overnight with primary antibodies, involving appropriately diluted CD34, VEGF and HIF-1 $\alpha$. Normal host serum was used for negative controls, followed by staining with appropriate HRP-conjugated secondary antibodies. The peroxidase was visualized with 3-3'-diaminobenzidinetetrahydrochloride solution and then counterstained with a weak hematoxylin solution stain. The stained slides were dehydrated and visualized on an Olympus microscope (Olympus, Japan). The stained sections were counted in the five areas of highest vascular density at $x 400$ magnification, and the MVD was expressed as the mean number of vessels in these areas.

Statistical analysis. Data are represented as mean \pm SD for the absolute values or percentage of the control. Statistical differences were evaluated by one-way analysis of variance (ANOVA) followed by LSD multiple comparison tests using SPSS software (version 17.0; SPSS, Inc.). A value of $\mathrm{P}<0.05$ was considered statistically significant.

\section{Results}

Analysis of miR-497 in breast cancer cell lines and clinical specimens. We examined the relative expression levels of miR-497 in several breast cancer cell lines, namely T-74D, MCF-7, MDA-MB-453, MDA-MB-468 and MDA-MB-435, along with MCF-10A, a non-malignant breast epithelial cell line, by RT-PCR. In all five breast cancer cell lines, the expression level of miR-497 was found lower than that in the MCF-10A cells (Fig. 1A). Since expression of miR-497 in the MCF-7 cells was much lower than the other four breast cancer cell lines, this cell line was used in the subsequent experiments. Next, we measured the miR-497 expression levels in 45 pairs of ductal breast cancer tissues and the corresponding adjacent non-cancerous tissues. Consistently, miR-497 was also downregulated in the breast cancer tissues in comparison with the level in the normal breast tissues (Fig. 1B). These results indicated that miR-497 expression was significantly suppressed in the breast cancer cell lines and tissue specimens.

Hypoxia inhibits the expression of $\mathrm{miR}-497$ in MCF-7 cells. First, the miR-497 expression in the MCF-7 cells was determined following transfection with miR-497 mimics, inhibitor or the negative control for $48 \mathrm{~h}$ by RT-PCR. Transfection with the miR-497 mimics resulted in a significant increase in its expression. Moreover, the miR-497 level was markedly decreased after the MCF-7 cells were transfected with the miR-497 inhibitor $(\mathrm{P}<0.05)$ (Fig. 2A). Afterwards, detection of miR-497 in the MCF-7 cells was performed by RT-PCR under normoxic and hypoxic conditions. It was found that the expression of miR-497 was significantly decreased under hypoxic conditions. In addition, the expression of miR-497 was increased after the MCF-7 cells were restored to a normoxic condition for $24 \mathrm{~h}$ (Fig. 2B).

Effects of conditioned medium on HUVEC proliferation and tube formation. Secondly, the effects of conditioned medium on HUVEC proliferation was evaluated. As shown in Fig. 3A, the CCK- 8 assay indicated that after being cultured in the conditioned medium from the miR-497 mimics group, the proliferation of HUVECs was relatively inhibited compared with that of the $\mathrm{NC}$ group $(\mathrm{P}<0.05)$. However, in comparison with the NC group, the miR-497 inhibitor increased the growth rate of HUVECs $(\mathrm{P}<0.05)$. In order to explore the effect of miR-497 on the capillary-like structure formation of HUVECs, an in vitro capillary tube formation assay was performed using the MCF-7 cell line. It was observed that the conditioned medium from the miR-497 mimics group displayed strong ability to inhibit the formation of capillarylike structures when compared to the NC group (Fig. 3B), 
A

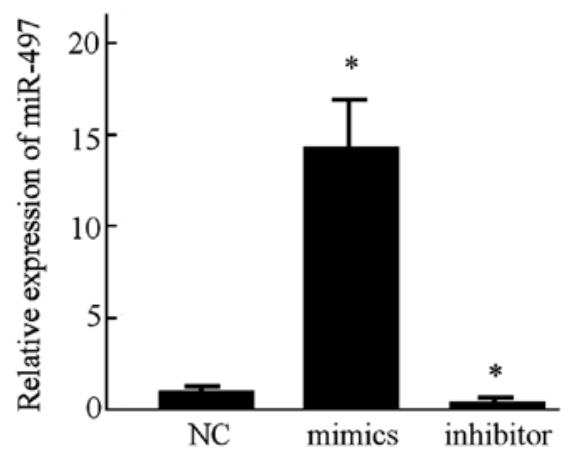

B

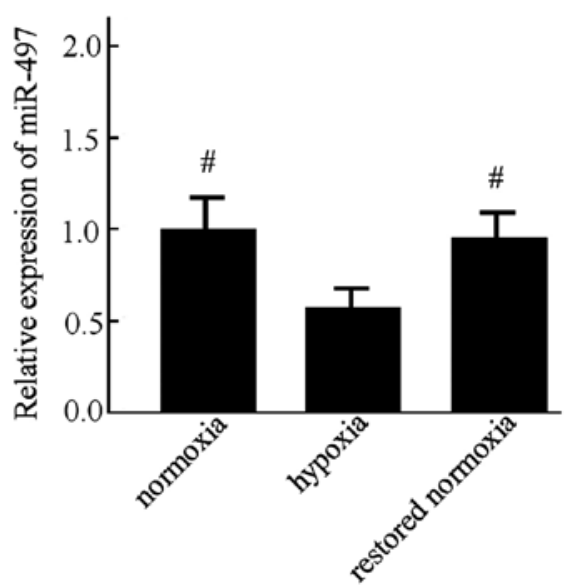

Figure 2. (A) The level of miR-497 in the NC group was lower than that in the miR-497 mimics group and higher than that in the miR-497 inhibitor group. (B) The expression of miR-497 in MCF-7 cells was detected by RT-PCR under normoxic and hypoxic conditions. ${ }^{*} \mathrm{P}<0.05$ vs. NC group, ${ }^{*} \mathrm{P}<0.05$ vs. hypoxic group.
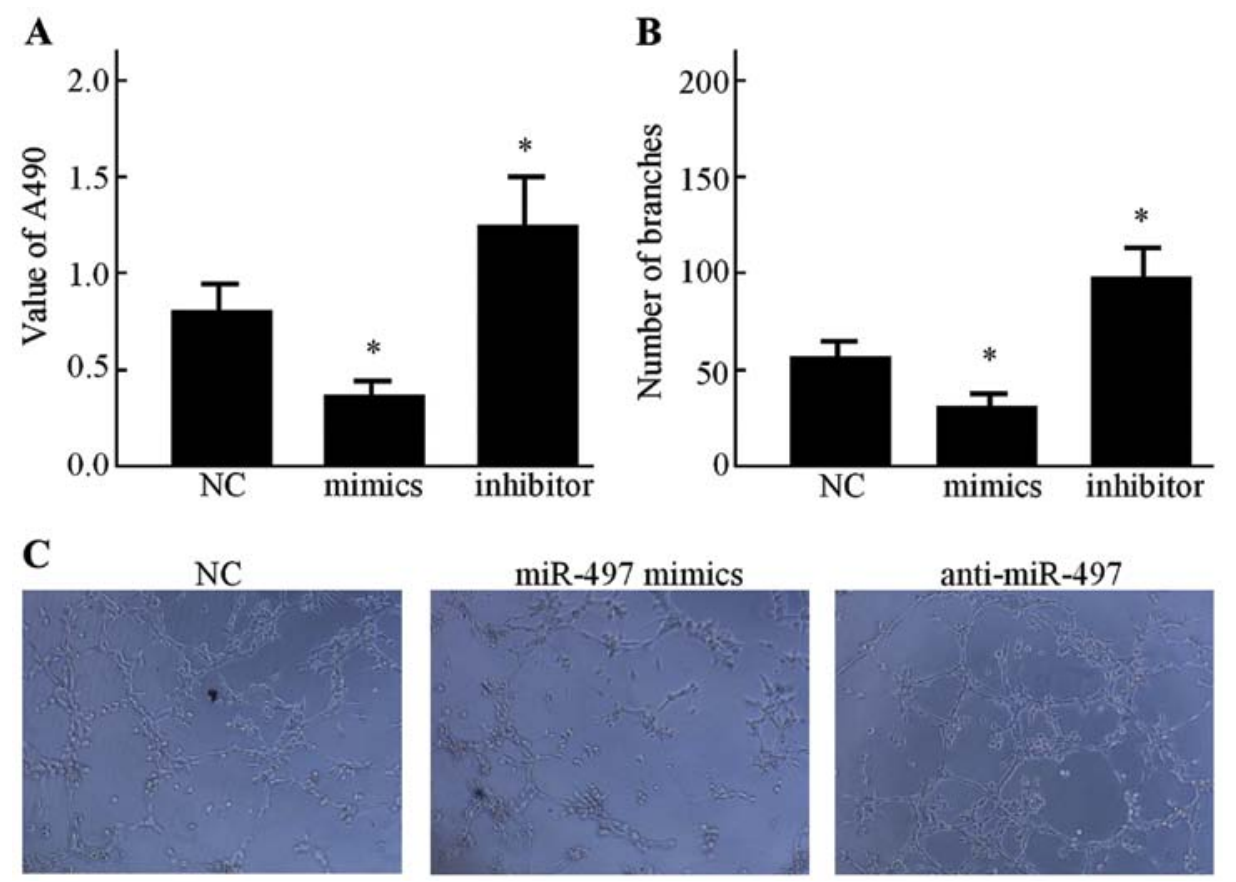

Figure 3. miR-497 exerts anti-proliferative and anti-angiogenic activity in vitro. (A) The proliferation of HUVECs by the conditioned media from the miR-497 mimics group was significantly inhibited compared with the NC group. (B) Assays of the capillary-like structure formation of HUVECs were performed by using conditioned media from the MCF-7 cells treated with miR-497 mimics, NC or anti-miR-497. (C) The relative images of endothelial tubule formation on Matrigel are shown at $\mathrm{x} 400$ magnification. ${ }^{*} \mathrm{P}<0.05$ vs. the $\mathrm{NC}$ group.

while the anti-miR-497 conditioned medium resulted in significant promotion of tubule formation of HUVECs on Matrigel (Fig. 3C).

$V E G F$ is the direct target of miR-497 and inhibits angiogenesis. To unveil the underlying mechanism of the disrupted angiogenesis mediated by miR-497, the expression of VEGF in MCF-7 cells was measured by western blot analysis. The result indicated that the overexpression of miR-497 reduced the protein level of VEGF while the suppression of miR-497 revealed an opposite result under normoxic conditions (Fig. 4A). Next, the relationship between miR-497 and
VEGF in conditions of normoxia or hypoxia was elucidated. As shown in Fig. 4B, in comparison with normoxia, hypoxia upregulated the expression of VEGF in the MCF-7 cells. Moreover, the VEGF expression was significantly decreased after the MCF-7 cells were restored to a normoxic condition. In addition, the MCF-7 cells were transfected with miR-497 mimics or anti-miR-497. The results revealed that VEGF secretion in the cell culture medium was markedly decreased in the MCF-7 cells that were transfected with the miR-497 mimics in a hypoxia condition (Fig. 4C). To further investigate the underlying mechanism, the expression of the transcription factor HIF-1 $\alpha$, which is responsible for VEGF regulation, in 
A

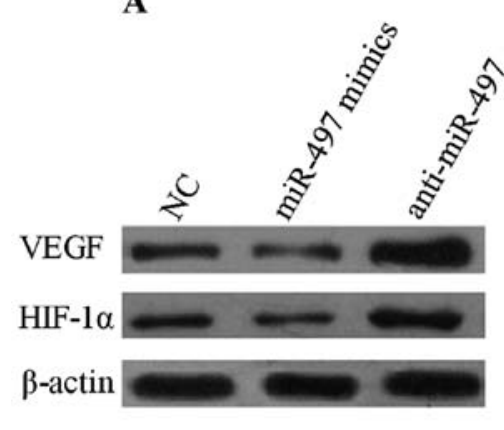

B
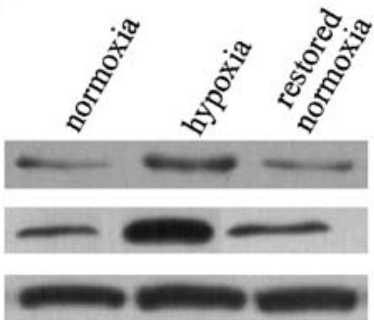

C

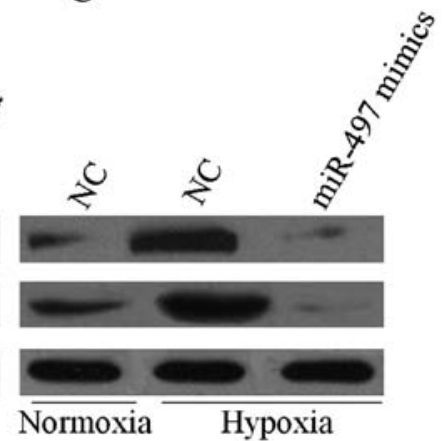

Figure 4. (A) Expression of VEGF and HIF-1 $\alpha$ were assessed by western blot analysis in the MCF-7 cells after transfection with miR497 mimics or anti-miR-497 under a normoxic condition. (B) The protein levels of VEGF and HIF-1 $\alpha$ in the MCF-7 cells under normoxia or hypoxia or restored to normoxia were detected by western blot analysis. (C) MCF-7 cells were transfected with miR-497 mimics or negative control under normoxic or hypoxic conditions.
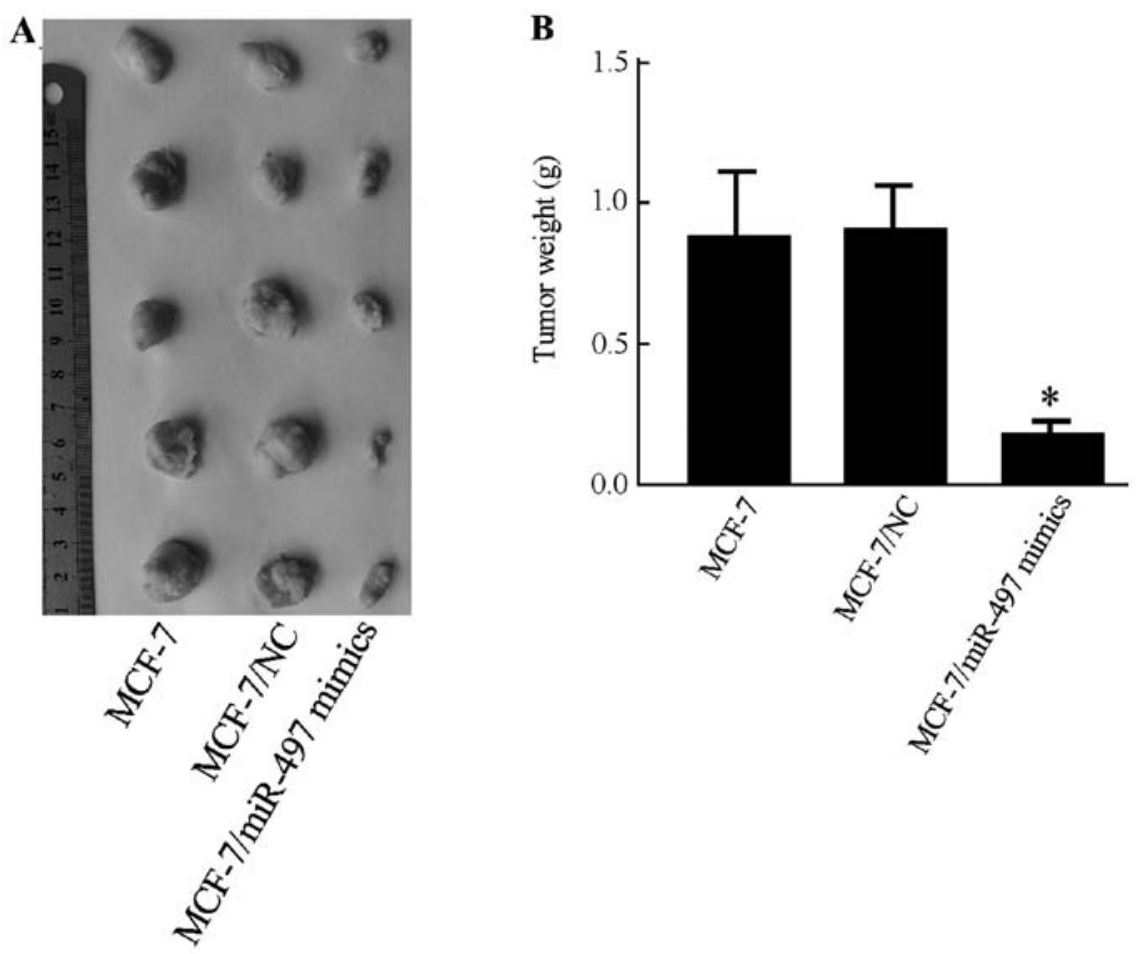

Figure 5. Effect of miR-497 on the in vivo growth of tumor xenografts. (A) Necropsy images of mouse tumors showing the therapeutic benefits of miR-497. (B) The mean weight of each tumor group. "P<0.05 vs. the MCF-7/NC group or the MCF-7 group.

MCF-7 cells was examined through western blot analysis. Fig. 4 shows that the expression of HIF-1 $\alpha$ was decreased in the MCF-7 cells after being transfected with the miR-497 mimics, correlating with decreased VEGF production.

miR-497 inhibits tumor growth in nude mice. To confirm the anti-tumorigenic effect of miR-497 in vivo, a xenograft model was performed to compare the tumorigenesis of MCF-7 cells before and after miR-497 transfection. Subcutaneous tumor nodes of different groups became palpable after 15 days of transplantation. The antitumor efficacy of miR-497 was determined by considering mean tumor weight immediately following euthanization. The final tumor weight showed a significant decrease in the MCF-7/miR-497 mimics group compared with MCF-7 or with MCF-7/NC groups $(\mathrm{P}<0.05)$ (Fig. 5A). The tumor weight in the MCF-7/NC group was not significantly different than that in the MCF-7 group $(\mathrm{P}=0.723)$ (Fig. 5B).

Tumor immunohistochemistry in vivo. As shown in Fig. 6, MCF-7 cells transfected with miR-497 mimics resulted in a marked reduction in vascularization microscopically compared with the negative control. In addition, comparatively, the microvascular density (MVD) was noticeably reduced in the MCF-7/ miR-497 mimics group. Subsequently, the expression of VEGF and HIF-1 $\alpha$ in the tumor tissues of all the three groups was examined by immunohistochemistry. Expression levels of VEGF and HIF-1 $\alpha$ were significantly downregulated in the tumor tissues of the MCF-7/miR-497 mimics group compared with these levels in the MCF-7 and MCF-7/NC groups, suggesting that miR-497 was directly involved in the inhibition of angiogenesis as well as tumor growth in nude mice. 

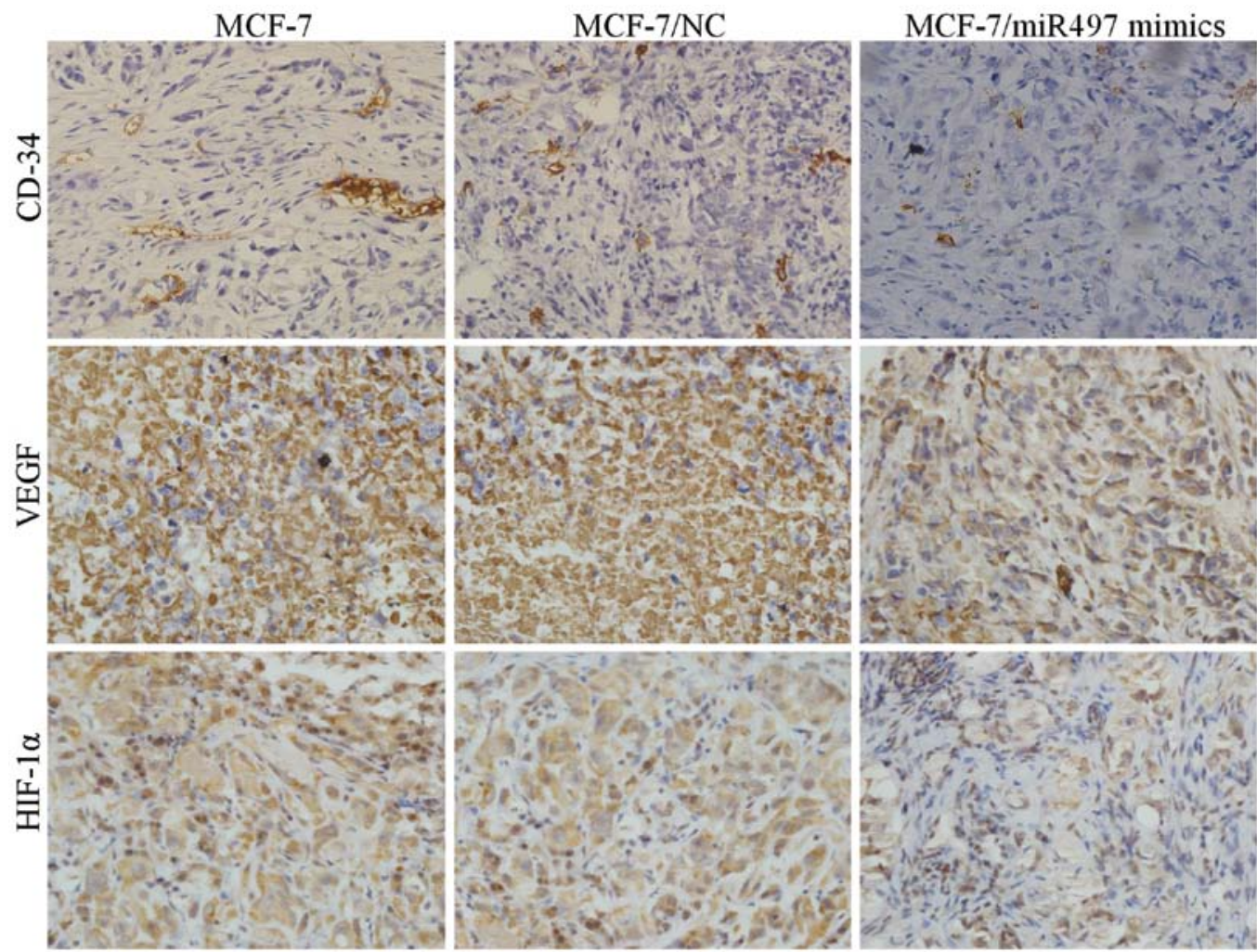

Figure 6. Immunohistochemistry for CD34, VEGF and HIF-1 $\alpha$ in the tumor tissue sections (original magnification, $\mathrm{x} 400$ ). Immunoreactivity towards all proteins were reduced in the MCF-7/miR-497 mimics group relative to their expression levels in the MCF-7/NC group or MCF-7 group.

\section{Discussion}

Solid tumors, such as breast carcinoma, are characterized by their heterogeneous nature, metastatic potential, regions of necrotic core and abnormal vasculature. Conventional treatments include surgery, immunization therapy and chemotherapy. Nevertheless, some patients who undergo chemotherapy experience early recurrence or metastasis, leading to poor prognosis $(4,5,17)$. The identification of new therapeutic targets for breast cancer treatment has become an intensive issue worldwide.

Over the past decades, research on the roles of miRNAs in the development of malignant tumors have been a 'hot' research topic. miRNAs are crucial players in many pathophysiological processes owing to their promising potential of being novel diagnostic and predictive markers for therapy. Currently, our insight into their roles in the development of solid tumors are rapidly accelerating (18). The majority of known and characterized miRNAs may function as tumor suppressors or oncogenes according to the cell type and tissue (19). Previous research demonstrated the tumor-suppressive role of miR-497 in several cancer types, including breast carcinoma (12-15). In the present study, our RT-PCR results indicated that the expression level of miR-497 in breast cancer tissues was markedly lower than that in corresponding adjacent non-cancerous tissues, which is consistent with a previous study (13). Moreover, our results showed that the miR-497 expression was also generally suppressed in all five breast cancer cell lines, in contrast to MCF-10A, which is a non-malignant breast epithelial cell line. Thus, we hypothesized that miR-497 may act as a valuable tumor suppressor in breast cancer and its functional mechanism warrants further investigation to support its significance. Accumulating evidence indicates that miR-497 induces apoptosis as well as regulates cell growth and invasion in breast cancer cells $(13,20)$. Yet, the potential role of miR-497 in angiogenesis remains unclear.

Recently, increasing evidence shows that angiogenesis is essential for the growth of solid tumors and research on tumor angiogenesis has become one of the most active fields in tumor treatment (21). Therefore, identifying anti-angiogenic targets is considered effective in tumor treatment. A recent study demonstrated angiogenesis-related markers in breast cancer (22). In the present study, we used the breast cancer cell line MCF-7 transfected with the miR-497 mimics to further investigate the effect of miR-497 on breast cancer-associated angiogenesis. Since it is well recognized that the process of tumor-derived angiogenesis is regulated by pro-angiogenic and anti-angiogenic factors that are secreted by tumor cells, we used the conditioned medium obtained from MCF-7 cells to eliminate the potential direct interaction between MCF-7 cells and HUVECs. Our results showed that the overexpression of miR-497 inhibited the in vitro proliferation of HUVECs and restrained angiogenesis in breast cancer cells by applying HUVECs. Hypoxia is considered an important factor in promoting angiogenesis, mainly by regulating proangiogenic and anti-angiogenic factors, such as VEGF (23). A previous study suggested that miR-210, as a hypoxia-inducible miRNA, augments the metastatic potential of liver cancer cells (24). Therefore, to validate the hypothesis that miR-497 can be induced by hypoxia, our results showed that the expression of miR-497 was significantly decreased under hypoxia. Additionally, as soon as the normoxic condition was restored, 
the level of miR-497 was immediately increased. Thus, the data suggested that hypoxia can reduce the expression of miR-497. To date, this is the first study to illuminate the dynamic change of miR-497 under normoxic and hypoxic conditions.

Furthermore, during the investigation of the mechanism of miR-497 in breast cancer cell progression, it was found that the overexpression of $\mathrm{miR}-497$ reduced the protein level of VEGF while suppression of miR-497 revealed an opposite result, and the expression of VEGF in the cell culture medium decreased markedly in the MCF-7 cells transfected with the miR-497 mimics, even under a hypoxia condition. In addition, our in vivo data disclosed that xenograft tumor tissues from the MCF-7/miR-497 mimics group displayed lower expression of CD34 and VEGF than the MCF-7 and MCF-7/NC groups. Previous research has demonstrated that the VEGF gene regulated by hypoxia is under the control of the transcription factor HIF-1, which consists of the hypoxic response factor HIF- $1 \alpha$ and the constitutively expressed aryl hydrocarbon receptor nuclear translocator HIF-1 $\beta / A R N T$ (25). Through the siRNA approach, the present study demonstrated that the overexpression of miR-497 inhibited the expression of HIF-1 $\alpha$, corresponding to changes in the expression of VEGF in vitro and in vivo. These results indicated that miR-497 exerts an anti-angiogenic effect by downregulating VEGF and HIF-1 $\alpha$ in breast cancer cells. Such data are consistent with those in the literature $(13,25)$.

In conclusion, our present findings are consistent with the hypothesis that miR-497 as a hypoxia-inducible miRNA, suppresses the angiogenesis of breast cancer cells in vitro as well as in vivo by inhibiting the expression of VEGF and HIF-1 $\alpha$. These findings suggest that hypoxia-related markers in breast cancer cells and the hypoxia/miR-497/HIF- $1 \alpha$ pathway may serve as a promising strategy for the treatment of breast cancer.

\section{References}

1. Torre LA, Bray F, Siegel RL, Ferlay J, Lortet-Tieulent J and Jemal A: Global cancer statistics, 2012. CA Cancer J Clin 65: 87-108, 2015.

2. Schulze A and Harris AL: How cancer metabolism is tuned for proliferation and vulnerable to disruption. Nature 491: 364-373, 2012.

3. Parks SK, Chiche J and Pouysségur J: Disrupting proton dynamics and energy metabolism for cancer therapy. Nat Rev Cancer 13: 611-623, 2013.

4. Zheng R, Yao Q, Xie G, Du S, Ren C, Wang Y and Yuan Y: TAT-ODD-p53 enhances the radiosensitivity of hypoxic breast cancer cells by inhibiting Parkin-mediated mitophagy. Oncotarget 6: 17417-17429, 2015.

5. Hamdan FH and Zihlif MA: Gene expression alterations in chronic hypoxic MCF7 breast cancer cell line. Genomics 104 477-481, 2014.

6. Soares RJ, Cagnin S, Chemello F, Silvestrin M, Musaro A, De Pitta C, Lanfranchi $G$ and Sandri M: Involvement of microRNAs in the regulation of muscle wasting during catabolic conditions. J Biol Chem 289: 21909-21925, 2014.

7. Godnic I, Zorc M, Jevsinek Skok D, Calin GA, Horvat S, Dove P, Kovac M and Kunej T: Genome-wide and species-wide in silico screening for intragenic MicroRNAs in human, mouse and chicken. PLoS One 8: e65165, 2013.
8. Gromak N, Dienstbier M, Macias S, Plass M, Eyras E, Cáceres JF and Proudfoot NJ: Drosha regulates gene expression independently of RNA cleavage function. Cell Rep 5: 1499-1510, 2013.

9. Zhai H, Chen QJ, Chen BD, Yang YN, Ma YT, Li XM, Liu F, Yu ZX, Xiang Y, Liao W, et al: Long noncoding RNA MALAT1 as a putative biomarker of lymph node metastasis: A meta-analysis. Int J Clin Exp Med 8: 7648-7654, 2015.

10. Nagpal N, Ahmad HM, Chameettachal S, Sundar D, Ghosh S and Kulshreshtha R: HIF-inducible miR-191 promotes migration in breast cancer through complex regulation of TGF $\beta$-signaling in hypoxic microenvironment. Sci Rep 5: 9650, 2015.

11. Chen W, Cai F, Zhang B, Barekati Z and Zhong XY: The level of circulating miRNA-10b and miRNA-373 in detecting lymph node metastasis of breast cancer: Potential biomarkers. Tumour Biol 34: 455-462, 2013.

12. Du M, Shi D, Yuan L, Li P, Chu H, Qin C, Yin C, Zhang Z and Wang M: Circulating miR-497 and miR-663b in plasma are potential novel biomarkers for bladder cancer. Sci Rep 5: 10437 , 2015.

13. Wei C, Luo Q, Sun X, Li D, Song H, Li X, Song J, Hua K and Fang L: microRNA-497 induces cell apoptosis by negatively regulating $\mathrm{Bcl}-2$ protein expression at the posttranscriptional level in human breast cancer. Int J Clin Exp Pathol 8: 7729-7739, 2015.

14. Troppan K, Wenzl K, Pichler M, Pursche B, Schwarzenbacher D, Feichtinger J, Thallinger GG, Beham-Schmid C, Neumeister P and Deutsch A: miR-199a and miR-497 are associated with better overall survival due to increased chemosensitivity in diffuse large B-cell lymphoma patients. Int J Mol Sci 16: 18077-18095, 2015.

15. Han Z, Zhang Y, Yang Q, Liu B, Wu J, Zhang Y, Yang C and Jiang Y: miR-497 and miR-34a retard lung cancer growth by co-inhibiting cyclin E1 (CCNE1). Oncotarget 6: 13149-13163, 2015.

16. Peng L, Liu A, Shen Y, Xu HZ, Yang SZ, Ying XZ, Liao W, Liu HX, Lin ZQ, Chen QY, et al: Antitumor and anti-angiogenesis effects of thymoquinone on osteosarcoma through the NF- $\kappa B$ pathway. Oncol Rep 29: 571-578, 2013.

17. Howell A, Anderson AS, Clarke RB, Duffy SW, Evans DG, Garcia-Closas M, Gescher AJ, Key TJ, Saxton JM and Harvie MN: Risk determination and prevention of breast cancer. Breast Cancer Res 16: 446, 2014.

18. Rinck A, Preusse M, Laggerbauer B, Lickert H, Engelhardt S and Theis FJ: The human transcriptome is enriched for miRNA-binding sites located in cooperativity-permitting distance. RNA Biol 10: 1125-1135, 2013.

19. Raychaudhuri S: MicroRNAs overexpressed in growth-restricted rat skeletal muscles regulate the glucose transport in cell culture targeting central TGF- $\beta$ factor SMAD4. PLoS One 7: e34596, 2012.

20. Luo Q, Li X, Gao Y, Long Y, Chen L, Huang Y and Fang L: MiRNA-497 regulates cell growth and invasion by targeting cyclin E1 in breast cancer. Cancer Cell Int 13: 95, 2013.

21. Liu Y, Wondimu A, Yan S, Bobb D and Ladisch S: Tumor gangliosides accelerate murine tumor angiogenesis. Angiogenesis 17: 563-571, 2014.

22. Retsky M, Bonadonna G, Demicheli R, Folkman J, Hrushesky W and Valagussa P: Hypothesis: Induced angiogenesis after surgery in premenopausal node-positive breast cancer patients is a major underlying reason why adjuvant chemotherapy works particularly well for those patients. Breast Cancer Res 6: R372-R374, 2004

23. Ma J, Zhang L, Ru GQ, Zhao ZS and Xu WJ: Upregulation of hypoxia inducible factor $1 \alpha$ mRNA is associated with elevated vascular endothelial growth factor expression and excessive angiogenesis and predicts a poor prognosis in gastric carcinoma. World J Gastroenterol 13: 1680-1686, 2007.

24. Dang K and Myers KA: The role of hypoxia-induced miR-210 in cancer progression. Int J Mol Sci 16: 6353-6372, 2015.

25. Meng F, Dong B, Li H, Fan D and Ding J: RNAi-mediated inhibition of Raf-1 leads to decreased angiogenesis and tumor growth in gastric cancer. Cancer Biol Ther 8: 174-179, 2009. 